\title{
Perylene-Diimide-Based Nanoparticles as Highly Efficient Photoacoustic Agents for Deep Brain Tumor Imaging in Living Mice
}

\section{Quli Fan,}

Key Laboratory for Organic Electronics \& Information Displays and Institute of Advanced Materials, Nanjing University of Posts \& Telecommunications Nanjing 210046, China

Molecular Imaging Program at Stanford (MIPS) Canary Center at Stanford for Cancer Early Detection Department of Radiology and Bio-X Program School of Medicine, Stanford University Stanford, California 94305-5484, USA

\section{Kai Cheng,}

Molecular Imaging Program at Stanford (MIPS) Canary Center at Stanford for Cancer Early Detection Department of Radiology and Bio-X Program School of Medicine, Stanford University Stanford, California 94305-5484, USA

\section{Zhen Yang,}

Key Laboratory for Organic Electronics \& Information Displays and Institute of Advanced Materials, Nanjing University of Posts \& Telecommunications Nanjing 210046, China

\section{Ruiping Zhang,}

Molecular Imaging Program at Stanford (MIPS) Canary Center at Stanford for Cancer Early Detection Department of Radiology and Bio-X Program School of Medicine, Stanford University Stanford, California 94305-5484, USA

Department of Radiology First Hospital of Shanxi Medical University Taiyuan 030001, China

\section{Min Yang,}

Molecular Imaging Program at Stanford (MIPS) Canary Center at Stanford for Cancer Early Detection Department of Radiology and Bio-X Program School of Medicine, Stanford University Stanford, California 94305-5484, USA

Key Laboratory of Nuclear Medicine Ministry of Health Jiangsu Key Laboratory of Molecular Nuclear Medicine Jiangsu Institute of Nuclear Medicine Wuxi 214063, China

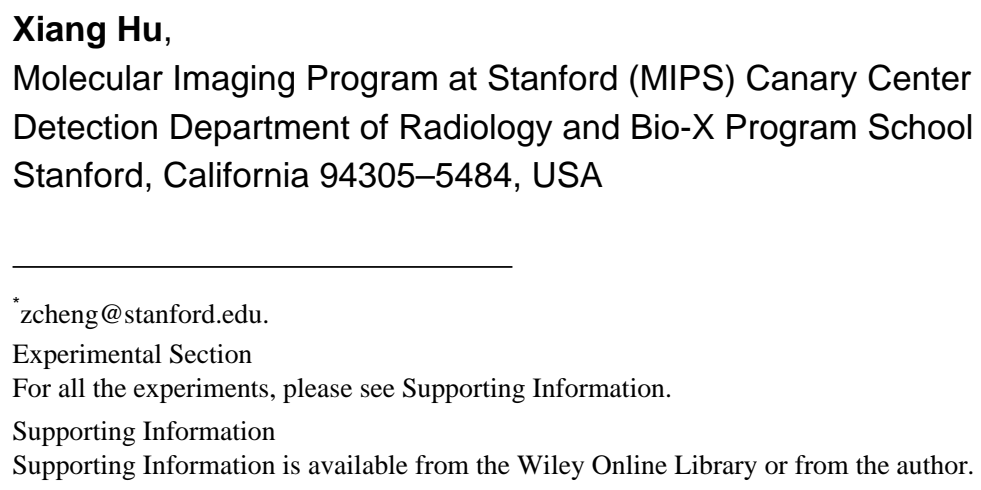

*zcheng@stanford.edu.

Experimental Section

For all the experiments, please see Supporting Information.

Supporting Information

Supporting Information is available from the Wiley Online Library or from the author. 
Xiaowei Ma,

Molecular Imaging Program at Stanford (MIPS) Canary Center at Stanford for Cancer Early Detection Department of Radiology and Bio-X Program School of Medicine, Stanford University Stanford, California 94305-5484, USA

Lihong Bu, Molecular Imaging Program at Stanford (MIPS) Canary Center at Stanford for Cancer Early Detection Department of Radiology and Bio-X Program School of Medicine, Stanford University Stanford, California 94305-5484, USA

Xiaomei Lu,

Key Laboratory for Organic Electronics \& Information Displays and Institute of Advanced Materials, Nanjing University of Posts \& Telecommunications Nanjing 210046, China

\section{Xiaoxing Xiong,}

Department of Neurosurgery School of Medicine Stanford University Stanford, California 94305, USA

Wei Huang,

Key Laboratory for Organic Electronics \& Information Displays and Institute of Advanced Materials, Nanjing University of Posts \& Telecommunications Nanjing 210046, China

Heng Zhao, and

Department of Neurosurgery School of Medicine Stanford University Stanford, California 94305, USA

\section{Zhen Cheng*}

Molecular Imaging Program at Stanford (MIPS) Canary Center at Stanford for Cancer Early Detection Department of Radiology and Bio-X Program School of Medicine, Stanford University Stanford, California 94305-5484, USA

Photoacoustic imaging (PAI) is a newly emerging technique in biomedical imaging that provides strong optical absorption contrast and high ultrasonic resolution, which could overcome the depth and resolution limits of conventional optical imaging techniques. ${ }^{[1]}$ Using near-infrared (NIR, 650-900 nm) absorptive materials as exogenous contrast agents has been widely applied because it improves PAI sensitivity and deep tissue penetration ability. With the rapid development of nanotechnology, numerous inorganic nanomaterials, such as gold, ${ }^{[2]}$ silver, ${ }^{[3]}$ copper ${ }^{[4]}$, and magnetic nanoparticles ${ }^{[5]}$ (NPs), and a few organic nanomaterials such as carbon nanotubes ${ }^{[6]}$ have been evaluated as PAI contrast agents. Many of these materials have also been conjugated with targeting moieties of interest and exhibited excellent PAI properties in vitro and in vivo. However, the nonbiodegradability and potential long-term toxicity of these nanomaterials severely hinder their clinical translation. More recently, conjugated polymer NPs with high NIR-absorption property ${ }^{[7]}$ have been developed as attractive PAI agents. But their poor biodegradability and metabolism in living body remains a problem for clinical use. In comparison, traditional NIR-absorptive and emissive small molecular organic dyes, such as indocyanine green (ICG) ${ }^{[8]}$ and other NIR dyes, ${ }^{[9]}$ have shown attractive properties for PAI. Especially, ICG has been approved by the Food and Drug Administration for patient use, highlighting the 
high clinical translation ability of organic dyes as imaging agents. However, the intrinsic optical instability, difficulty of modification, and high cost of those organic dyes dramatically limit their applications.

Despite of the good PAI property of the aforementioned materials, realizing their capability for deep tissue detection is a big challenge. Although carefully choosing the excitation NIR laser wavelength can efficiently enhance the detection depth, the light scattering in different tissues, and the absorption by endogenous hemoglobin in blood drastically reduce the laser flux to the deep region. For example, the physiological features of superficial brain tissues have been widely investigated using PAI contrast agents, ${ }^{[4 a, 6 a, 10]}$ while only most recently silica-coated Au NPs were used for PA of superficial glioma in mouse models. [11,12] However, in vivo PAI of deep brain tumor is still difficult (the laser flux will be further reduced by the skull). Therefore, exploring novel biomaterials for PAI to collect more accurate information of deep tumor is highly desired.

Perylene-3,4,9,10-tetracarboxylic diimide (PDI) and its derivatives are a class of small molecules that possess exceptional high chemical, thermal, and photochemical stabilities, good optoelectronic property, easy modification, and extremely low cost. Due to their excellent performance, they have been widely developed as prominent materials for constructing various organic electronic devices. ${ }^{[13]}$ In this study, for the first time, we successfully developed the PDI-based NIR-absorptive organic NPs (micelle-enveloped PDI) as an efficient contrast agent for PAI of deep brain tumor in living mice. By encapsulating into amphiphilic molecules, water-soluble PDI NPs were easily synthesized and exhibited excellent PAI property (Figure 1 a). We further demonstrated that the NPs were efficiently used for detection of mouse brain tumor with high PAI contrast in vivo. The unique properties of PDI with high biocompatibility, high photostability, and PAI sensitivity warrant it as a novel biomaterial for broad imaging applications.

PDI molecule structured with tertiary amine group (electron donor) and diimide group (electron acceptor) to form traditional donor- $\pi$-acceptor system for enhancing bathochromic effect was easily modified to realize its NIR absorption (Figures S1-S4, Supporting Information). Water-soluble PDI NPs (Figure $1 \mathrm{~b}$ ) were then obtained through enveloping hydrophobic PDI with amphiphilic DSPE-mPEG5000. The NPs prepared showed high monodispersity and homogeneity with the similar measured average particle size of $48.0 \mathrm{~nm}$ (by transmission electron microscopy (TEM), Figure $1 \mathrm{c}$ ) and $53.0 \mathrm{~nm}$ (by dynamic light scattering, Figure $1 \mathrm{~d}$ ), respectively. The PDI NPs exhibited NIR absorption in aqueous solution with a maximum absorption of around $700 \mathrm{~nm}$ as shown in Figure $1 \mathrm{e}$ (detailed discussions of the optical properties of PDI dye were showed in Figures S5-S7 and Table $\mathrm{S} 1$, Supporting Information). The conjugated number of PDI molecules per NP was calculated to be about $1.0 \times 10^{4}$, and the extinction coefficient of PDI NP at $700 \mathrm{~nm}$ was $2.00 \times 10^{8} \mathrm{M}^{-1} \mathrm{~cm}^{-1}$, suggesting it is an excellent NIR light absorber (see Figures S8 and S9, Supporting Information).

The as-prepared PDI NPs were highly stable and can be stored in phosphate-buffered saline (PBS, $0.1 \mathrm{~m}, \mathrm{pH}=7.4$ ) without any precipitation in the refrigerator for at least two months. No PDI molecule leaching out of the NPs was observed after incubation for $48 \mathrm{~h}$ in mouse 
serum at $37{ }^{\circ} \mathrm{C}$ (see Figure S10, Supporting Information). More importantly, compared with commercial ICG that exhibited considerably reduced absorption (>80\%) under light exposure, PDI NPs were found to be intriguing photostability (only $5 \%$ reduced absorption) after $60 \mathrm{~min}$ of continuous laser irradiation at $700 \mathrm{~nm}$ and $8 \mathrm{~mJ} \mathrm{~cm}^{-2}$ (Figure $1 \mathrm{f}$ ). This result highlights the outstanding optical stability of the obtained PDI NPs, which is a significant advantage of contrast agents for PAI.

For PAI application in vivo, the detection sensitivity of PDI NPs in living body was then tested by subcutaneous injection of PDI NPs on the lower back of mice. Their ultrasound (US) and PA images were collected (Figure S11a, Supporting Information) and showed that the PA signal decreases linearly with the concentration decrease $\left(R^{2}=0.990\right.$, Figure S11b, Supporting Information). Furthermore, $0.625 \mathrm{nM}$ of PDI NPs was found to give the equivalent PA signal strength as the tissue background, highlighting the high detection sensitivity of PDI-based PA imaging technique.

To examine the imaging performance of PDI NPs in brain tumor in vivo, the orthotopic glioblastoma tumor models by implanting C6-Fluc cells and the control models by injection of PBS in the mice brains were established. Bioluminescence imaging was used to monitor the tumor growth, and magnetic resonance imaging (MRI) providing anatomical information was also used to accurately localize the tumors (the region in yellow circle in Figure S12, Supporting Information). After tail vein injection of PDI NPs, the coronal section of PAI was collected for investigating the PAI depth in our system. In Figure 2a, a weak PA signal above the skull region was observed both in tumor mice and control mice before NPs injection which may be derived from the capillary vessels in the skin and the trauma on the skull and skin under surgery. After $2 \mathrm{~h}$ injection, the PA imaging at the same region became much brighter because of the existence of circulating PDI NPs in the blood vessel. At the same time, no PA signal was found in the brain. After $1 \mathrm{~d}$ injection, an obvious PA signal appeared in the brain of tumor mice whereas no signal was found in the control mice. Such PA position in the mouse brain was in accordance with the tumor position obtained from MRI, demonstrating excellent PAI effect of PDI NPs on brain tumor. PDI NPs were anticipated to enter the extravascular space by diffusion through the disrupted blood brain barrier as a result of the tumor formation and accumulation in the tumor cells. Such enhanced permeability and retention (EPR) effect thus contributed to the enhanced PAI signal at the orthotopic tumor sites, which has been reported for many inorganic NPs. ${ }^{[14]}$

After $2 \mathrm{~d}$ injection, a deeper tumor region (depth at $4.0 \mathrm{~mm}$ ) was more clearly visualized from PAI (Figure $2 \mathrm{a}$ ) than after $1 \mathrm{~d}$ injection (depth at $3.0 \mathrm{~mm}$ ). The enhanced tumor penetration of NPs with time was generally used to explain this phenomenon. Compared with the solid silica-coated Au NPs with the size of larger than $100 \mathrm{~nm}$ used for brain tumor detection, ${ }^{[10]}$ the relatively smaller size (about $50 \mathrm{~nm}$ ) and softer structure of the organic PDI NPs may achieve better tumor penetration ability to obtain deep tumor PAI. ${ }^{[15]}$ It is intriguing to find that the increase of detectable tumor depth was accompanied with the decreased PA signals above the skull (in red dotted circle) and in the tumor region (in blue dotted circle) with time (Figure $2 \mathrm{~b}, 2 \mathrm{~d}$ vs $1 \mathrm{~d}$ ). Besides the enhanced tumor penetration of NPs, the depth of tumor PAI was also determined by the laser flux to the deep tumor region. Considering the limitation of laser energy safely used for in vivo imaging, the decreased 
laser absorption above the skull at $2 \mathrm{~d}$ in our system provided the enhanced laser photon flux to the tumor region which could enhance the tumor PAI. Thereafter, the laser penetration in tumor should contribute to the PAI depth effect of tumor. Due to the existence of PA contrast agent in tumor, the laser flux to the deep tumor region and the resultant PA signal were assumed to be determined by the absorption property and NPs concentration in tumor while, to our knowledge, no related work was reported. Because of its difficulty to be investigated in vivo, we built up an in vivo phantom to try to disclose the NP concentrationPA depth relationship to some extent. The agarose gels with the same volume buried with different concentrations of PDI NPs were used to mimic the tumor region with different NPs contents, and their PA signals were measured and compared with each other (Figure $2 \mathrm{~d}, \mathrm{e}$ ). Our results of phantom study clearly demonstrated that lower flux of laser light was transmitted to the deeper region at high NPs concentrations because of the absorption of most excitation light by the NPs close to the surface. With the decrease of NPs concentration, more light could penetrate through the surface to the deep location, therefore achieving more detectable PA depth although the PA signal still decreased. Thus, based on the similarity of the tumor PAI with the phantom study, the enhanced detectable tumor depth by PAI at $2 \mathrm{~d}$ may also result from the decreased NP concentration in the superficial region of tumor to rend more laser flux to the deep region. It should be emphasized that because of the weak laser energy transferred to the deep region, the strong absorption property of PA agents is no doubtfully very essential for PAI. ${ }^{[11]}$ However, overstrong absorption of the contrast agents may lead to the undesired increased light absorption at the superficial regions of the tumor and thus reduce the production of PA signal from the deeper region. This may explain why the silicon-coated Au NPs with high sensitivity $\left(10^{9} \mathrm{~cm}^{-1} \mathrm{M}^{-1}\right)$ looks only produce the PA signal at the superficial region of the tumor (no PA depth data provided) ${ }^{[11]}$, whereas PDI NPs with relative low absorption $\left(10^{8} \mathrm{~cm}^{-1} \mathrm{M}^{-1}\right)$ could provide deeper PA information.

The localization of PDI NPs in brain tumor was further confirmed by PA spectra measurements. In Figure $2 \mathrm{c}$, it can be clearly seen that the tumor region after $2 \mathrm{~d}$ NPs injection exhibited the strong peaks of PA signal at 700 and $735 \mathrm{~nm}$ (blue line). This observation is in accordance with the NIR absorption peak of PDI NPs (Figure $1 \mathrm{e}$ ) and the PA peaks of pure PDI NPs in aqueous solution (black line) while much different from the PA peaks before NP injection which is from the typical endogenous hemo-oxygen in blood (red line), indicating the successful accumulation of PDI NPs in the tumor. Interestingly, different with PDI NPs in aqueous solution with the maximum PA peak at $700 \mathrm{~nm}$, the maximum PA peak at $760 \mathrm{~nm}$ was found in tumor region. It is conceivable that besides the absorption capability of NPs, the penetrability of the excited laser at different wavelength also highly influenced the PA signal in the deep region.

To diminish the interference PA signal around the skull region and further testify the capability of PDI NPs for deep tumor PAI, ex vivo US and PA imagings of mice brains which were harvested and buried in agarose gel were studied to compare with the in vivo imaging. In Figure 3a, the ex vivo PAI quality of brain tumor was obviously more clear than in vivo, and the accumulation of PDI NPs in the brain was evident from the PA signal. In contrast, no PA signal was detected in the mouse brain injected with PBS. Since the 
similar position of PA signal in the brain of tumor mouse ex vivo with the tumor MRI in vivo, the PDI NPs exhibited favorable property as a PA contrast agent for in vivo deep brain tumor detection simply by EPR effect. Furthermore, the spatial accumulation and distribution of PDI NPs in tumor can be well mapped through constructing the 3D US and PA image (Figure $3 \mathrm{~b}$ ). It was showed that the NPs localized around the spatial position to which the C6-Fluc cells were injected, indicating the excellent 3D PAI quality of PDI NPs for tumor imaging.

The NP clearance and biodistribution at $2 \mathrm{~d}$ were investigated by ex vivo PA signal quantification of the resected organs embedded in agarose gel (Figure S13a, Supporting Information). The biodistribution in the mice injected with PBS was also analyzed for comparison. It was found that very low PA signal presented in all organs for the mice injected with PBS. As for the mice injected with PDI NPs, the NPs exhibited dominant uptake in the liver and the spleen, a significantly lower uptake in the kidney, skin, intestine, stomach, and heart, and nearly no accumulation in the bone, pancreas, and muscle (Figure S13b, Supporting Information). This distribution pattern is quite similar to those reported for inorganic and organic NPs because the NPs with size higher than $10 \mathrm{~nm}$ are mainly cleared through hepatobiliary system. ${ }^{[16]}$

The relative high viability of NIH3T3 cells (95\%-105\% as compared with the nontoxic control) after $24 \mathrm{~h}$ of incubation with different concentrations of PDI NPs indicated their good biocompatibility and low cytotoxic effect (Figure S14a, Supporting Information). The in vivo toxicity of PDI NPs in mice was further evaluated through H\&E staining of those organs in which the NPs highly accumulated. Following tail vein injection of PDI NPs, there was no death or abnormal behavior in any of the experimental animal groups during the study period. H\&E staining of the liver, the spleen, and the kidneys did not show any apparent changes in cellular structures after $7 \mathrm{~d}$ NPs injection (Figure S14b, Supporting Information). These preliminary results demonstrated that intravenous administration of PDI NPs did not cause acute toxicity to the liver, the spleen, or the kidney in mice. Further investigations are still needed to comprehensively evaluate the NPs toxicity.

In conclusion, PDI NPs were successfully prepared and used to realize PAI of deep orthotopic brain tumor in mice models. Because of its low cost, ease of chemical modifications, and extraordinary high photostability, PDI system exhibits high potential for PAI of deep tumor. Furthermore, selecting contrast agents with appropriate light absorption capability and/or controlling their concentrations in tumor in an appropriate extent may be essential to obtain good PAI of deep tumor. Overall, our work provides insight on how to design or choose appropriate organic NP-based contrast agents for promoting the development of PAI technology for deep tumor imaging.

\section{Supplementary Material}

Refer to Web version on PubMed Central for supplementary material. 


\section{Acknowledgements}

Q.F. and K.C. contributed equally to this work. This work was supported, in part, by the Offi ce of Science (BER), the U.S. Department of Energy (DE-SC0008397), NCI of Cancer Nanotechnology Excellence Grant CCNE-TR U54 CA119367, CA151459, NIH In vivo Cellular Molecular Imaging Center (ICMIC) grant P50 CA114747, the National Basic Research Program of China (No. 2012CB723402), and the National Natural Science Foundation of China (Nos. 21222404, 61378081, 81171399, 2011832173, 2012ZX09505-001-001, RC2011095, NY211003).

\section{References}

1. Wang LV, Hu S. Science. 2012; 335:1458. [PubMed: 22442475]

2. a Song KH, Kim C, Cobley CM, Xia Y, Wang LV. Nano Lett. 2009; 9:183. [PubMed: 19072058] b Jokerst JV, Thangaraj M, Kempen PJ, Sinclair R, Gambhir SS. ACS Nano. 2012; 6:5920. [PubMed: 22681633] c Cheng K, Kothapalli SR, Liu H, Koh AL, Jokerst JV, Jiang H, Yang M, Li J, Levi J, Wu JC, Gambhir SS, Cheng Z. J. Am. Chem. Soc. 2014; 136:3560. [PubMed: 24495038]

3. Homan KA, Souza M, Truby R, Luke GP, Green C, Vreeland E, Emelianov S. ACS Nano. 2012; 6:641. [PubMed: 22188516]

4. Ku G, Zhou M, Song S, Huang Q, Hazle J, Li C. ACS Nano. 2012; 6:7489. [PubMed: 22812694]

5. a Hao R, Xing RJ, Xu ZC, Hou YL, Gao S, Sun SH. Adv. Mater. 2010; 22:2729. [PubMed: 20473985] b Yu J, Yang C, Li JDS, Ding YC, Zhang L, Yousaf MZ, Li J, Pang R, Wei LB, Xu LL, Sheng FG, Li CH, Li GJ, Zhao LY, Hou YL. Adv. Mater. 2014; 26:4114. [PubMed: 24677251]

6. De la Zerda A, Zavaleta C, Keren S, Vaithilingam S, Bodapati S, Liu Z, Levi J, Smith BR, Ma TJ, Oralkan O, Cheng Z, Chen X, Dai H, Khuri-Yakub BT, Gambhir SS. Nat. Nanotechnol. 2008; 3:557. [PubMed: 18772918]

7. a Zha Z, Deng Z, Li Y, Li C, Wang J, Wang S, Qu E, Dai Z. Nanoscale. 2013; 5:4462. [PubMed: 23584573] b Pu K, Shuhendler AJ, Jokerst JV, Mei J, Gambhir SS, Bao Z, Rao J. Nat. Nanotechnol. 2014; 9:233. [PubMed: 24463363] c Liu J, Geng J, Liao LD, Thakor N, Gao X, Liu B. Polym. Chem. 2014; 5:2854.

8. Kim C, Song KH, Gao F, Wang LV. Radiology. 2010; 255:442. [PubMed: 20413757]

9. a Akers WJ, Kim C, Berezin M, Guo K, Fuhrhop R, Lanza GM, Fischer GM, Daltrozzo E, Zumbusch A, Cai X, Wang LV, Achilefu S. ACS Nano. 2011; 5:173. [PubMed: 21171567] b de la Zerda A, Bodapati S, Teed R, May SY, Tabakman SM, Liu Z, Khuri-Yakub BT, Chen X, Dai H, Gambhir SS. ACS Nano. 2012; 6:4694. [PubMed: 22607191]

10. a Yang X, Skrabalak SE, Li ZY, Xia Y, Wang LV. Nano Lett. 2007; 7:3798. [PubMed: 18020475] b Lu W, Huang Q, Ku G, Wen X, Zhou M, Guzatov D, Brecht P, Su R, Oraevsky A, Wang LV, Li C. Biomaterials. 2010; 31:2617. [PubMed: 20036000]

11. Kircher MF, de la Zerda A, Jokerst JV, Zavaleta CL, Kempen PJ, Mittra E, Pitter K, Huang R, Campos C, Habte F, Sinclair R, Brennan CW, Mellinghoff IK, Holland EC, Gambhir SS. Nat. Mater. 2012; 18:829.

12. Lu W, Melancon MP, Xiong C, Huang Q, Elliott A, Song S, Zhang R, Flores LG, Gelovani JG, Wang LV, Ku G, Stafford RJ, Li C. Cancer Res. 2011; 71:6116. [PubMed: 21856744]

13. a Lindner SM, Huttner S, Chiche A, Thelakkat M, Krausch G. Angew. Chem. 2006; 118:3442.Angew. Chem. Int. Ed. 2006; 45:3364.b Liu Y, Wang KR, Guo DS, Jiang BP. Adv. Funct. Mater. 2009; 19:2230.c Ohta T, Nagano T, Ochi K, Kubozono Y, Shikoh E, Fujiwara A. Appl. Phys. Lett. 2006; 89:053508.

14. Trehin R, Figueiredo JL, Pittet MJ, Weissleder R, Josephson L, Mahmood U. Neoplasia. 2006; 8:302. [PubMed: 16756722]

15. Waite CL, Roth CM. Crit. Rev. Bioeng. 2012; 40:21.

16. Longmire M, Choyke PL, Kobayashi H. Nanomedicine. 2008; 3:703. [PubMed: 18817471] 
a
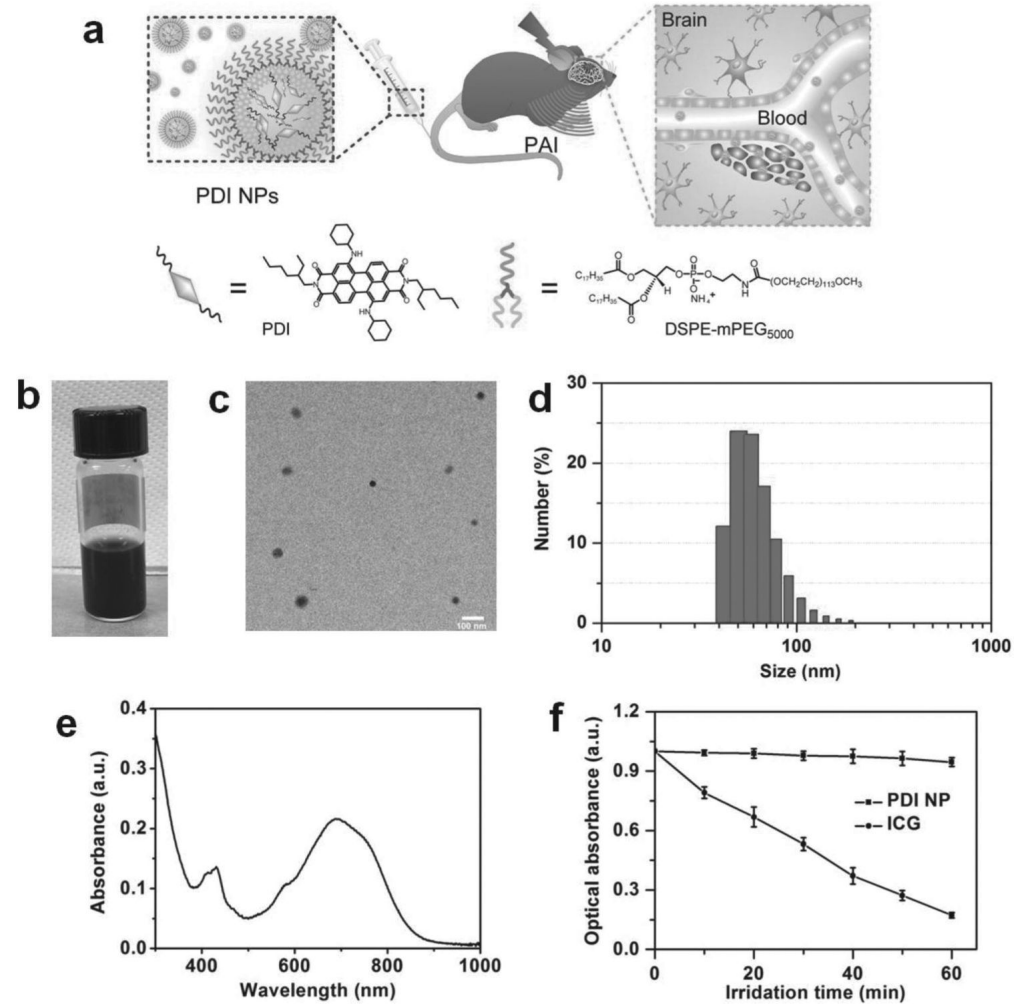

Figure 1.

a) Schematically illustrating the PAI process of brain tumor in vivo by PDI NPs. b) Picture of PDI NPs in PBS ( $\mathrm{pH}=7.4)$. c) TEM of PDI NPs $(\mathrm{Bar}=100 \mathrm{~nm})$. d) Hydrodynamic size distribution graph of PDI NPs. e) UV-vis-NIR absorption spectrum of PDI NPs in aqueous solution. f) Photostability comparison of PDI NPs with ICG. 

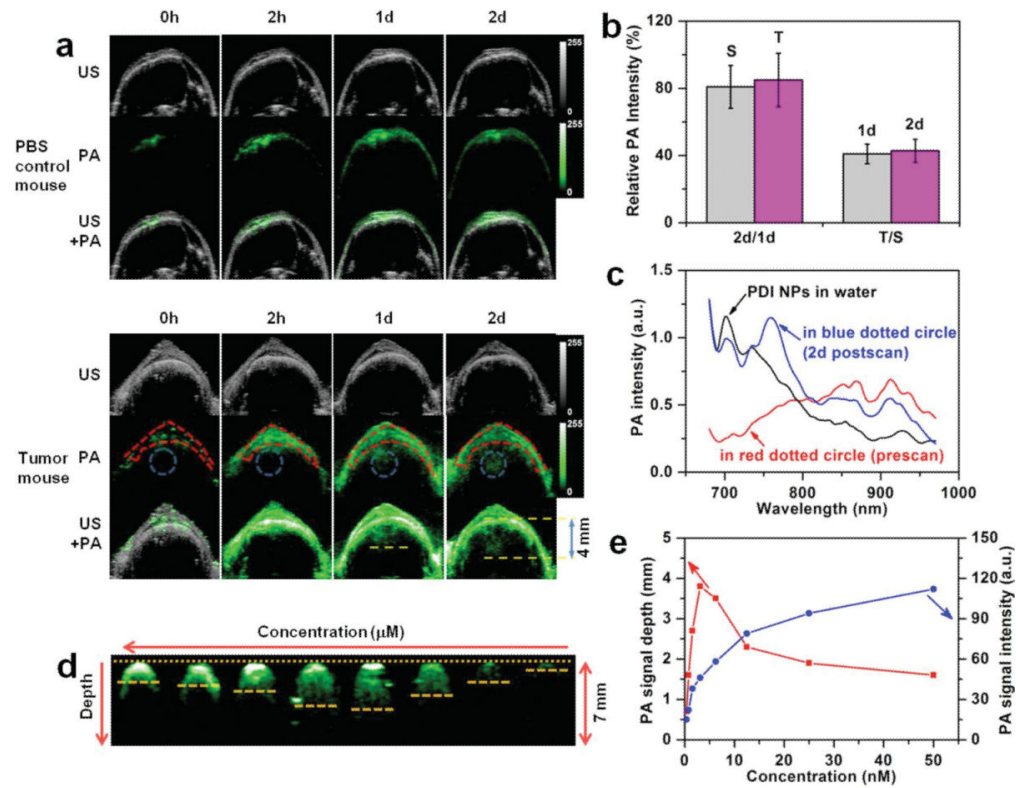

Figure 2.

a) The US (grey), PA (green), and their overlay coronal sections of brain of control model (top) and tumor model (bottom) before and after tail vein injection of $250 \mu \mathrm{L}$ of $250 \mathrm{nM}$ PDI NPs. b) The relative PA signal changes of the skull region ( $\mathbf{S}$, in red dotted circle of (a)) and tumor region ( $\mathbf{T}$, in blue dotted circle of (a)) at $2 \mathrm{~d}$ compared with that at $1 \mathrm{~d}$, respectively, and that of $\mathbf{T} / \mathbf{S}$ at $1 \mathrm{~d}$ and $2 \mathrm{~d}$. c) PA spectra of PDI NPs in aqueous solution (black line), the region in red dotted circle of (a) before injection of NPs (red line) and tumor region of (a) after $2 \mathrm{~d}$ injection of NPs (blue line). d) The PA coronal sections of PDI NPs with different concentrations (from 50 to $0.390625 \mathrm{nM}$ ) in agarose gel (in vivo phantom). e) The plots of PA intensity-NP concentration and PA detectable depth-NP concentation relationships. 


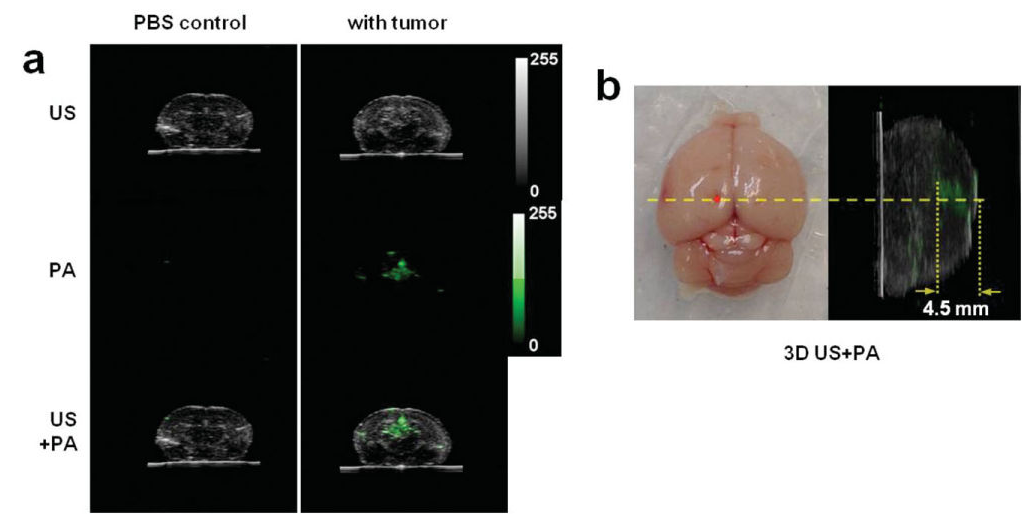

Figure 3.

a) The ultrasonic (grey), photoacoustic (green), and their overlay coronal sections of brain of control model (left) and tumor model (right) which were harvested and buried in agarose gel after $2 \mathrm{~d}$ tail vein injection of PDI NPs. b) Photographic transverse imaging (left) and the overlay of PA (green) and US (grey) sagittal 3D image (right) of tumor bearing brain. The red dot in fi gure (b) is the injection point of C6-Fluc cell. 